\title{
Detection of Coherent Oceanic Structures via Transfer Operators
}

\author{
Gary Froyland ${ }^{1,{ }^{*}}$, Kathrin Padberg ${ }^{2}$, Matthew H. England ${ }^{1}$ and Anne Marie Treguier $^{3}$ \\ ${ }^{1}$ School of Mathematics and Statistics, The University of New South Wales, Sydney NSW 2052, Australia \\ ${ }^{2}$ Fakultät für Elektrotechnik, Informatik und Mathematik, Universität Paderborn, 33095 Paderborn, Germany \\ ${ }^{3}$ CNRS, IFREMER, LPO B.P.70, 29280 Plouzane, France \\ *: Corresponding author : G. Froyland, email address : g.froyland@unsw.edu.au
}

\begin{abstract}
:
Coherent nondispersive structures are known to play a crucial role in explaining transport in nonautonomous dynamical systems such as ocean flows. These structures are difficult to extract from model output as they are Lagrangian by nature and not revealed by the underlying Eulerian velocity fields. In the last few years heuristic concepts such as finite-time Lyapunov exponents have been used in an attempt to detect barriers to oceanic transport and thus identify regions that trap material such as nutrients and phytoplankton. In this Letter we pursue a novel, more direct approach to uncover coherent regions in the surface ocean using high-resolution model velocity data. Our method is based upon numerically constructing a transfer operator that controls the surface transport of particles over a short period. We apply our technique to the polar latitudes of the Southern Ocean.
\end{abstract}

\section{INTRODUCTION}

The rate and pathways of horizontal dispersion in the ocean are of great importance for many problems, including the transport of biomass and pollutants, and the detection of filaments and stirring. Coherent structures, such as gyres and eddies, can house low-dispersion regions where biomass can be trapped over long periods. These persistent non-dispersive regions are known to play a crucial role in oceanic circulation as they act as transport barriers. While persistent features such as gyres and eddies may be observed and tracked by satellite altimetry [1], detecting and tracking the regions that act as barriers to Lagrangian flow pathways is more ambiguous. This is true even if the surface velocity field is perfectly known. For example, mathematical models of the global ocean circulation can currently be constructed at a 1/12th degree resolution and satellite data and float velocity measurements can be used to estimate flow fields in the real system. However, it is difficult to transform these modeled or observed velocity fields into a description of coherent structures or low dispersion areas. In order to assess the mathematical models or observations at increasing resolution and complexity, it is important to develop efficient numerical methods to describe the predicted coherent structures and dispersion. A commonly used approach for this problem is to plot a timeaveraged vector field and eyeball this field to obtain an estimate of where coherent structures lie. A drawback to this method is that these structures are often Lagrangian in nature and thus do not show up in an (averaged) Eulerian velocity field. Another approach uses finite-time Lyapunov exponents (FTLEs), which quantify the local rate of separation of model trajectories. Theory [2-4] suggests that peaks of the FTLE field correspond to finite-time invariant man- ifolds: curves or surfaces that are 
approximately invariant for a short time. These finite-time invariant manifolds represent barriers to transport, as trajectories are very unlikely to cross them [4]. The regions enclosed by these objects form time-dependent persistent structures that trap seawater, biomass, and nutrients. FTLE and other dynamical systems approaches have had success in analysing oceanic flows, see [5] and references therein, but they have not addressed the detection of large, min- imally dispersive structures at the oceanic scale, nor the quantification of the extent to which mass is trapped by these structures. Moreover, these concepts may not even be able to explain all transport mechanisms at work [6].

The purpose of this note is to describe a new, more direct method of identifying these coherent structures in the surface ocean. We will test this approach in the context of the high latitude Southern Ocean, a region low in measurements but important for climatic and biological applications. Our method is based upon numerically constructing a transfer operator that controls the horizontal ocean circulation from a time $t$ to a short time later $t+\tau$. The eigenfunctions of this transfer operator corresponding to large positive eigenvalues directly reveal dominant "almost-invariant" structures in the surface flow over the time period considered. These structures retain their shape over the period $[t, t+\tau]$ and thus "trap" most of the water inside them with only minimal leakage. In addition, our approach allows us to quantify the mass leakage of the identified regions. 


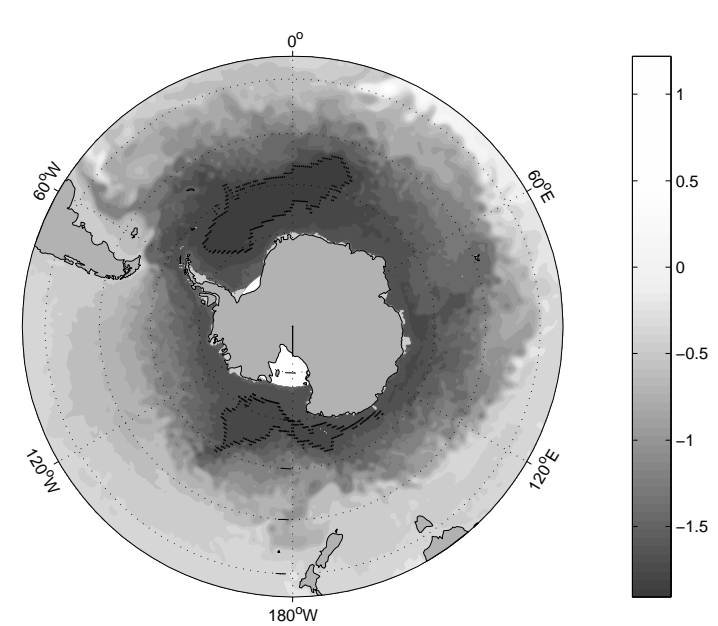

FIG. 1: Mean SSH (m) from ORCA025 model averaged over 1 January-29 February. Define regions $A_{\text {Weddell,SSH }}=\{\mathrm{SSH}<-1.75 \mathrm{~m}$ in Weddell Sea $\}$ and $A_{\text {Ross }, \mathrm{SSH}}=\{\mathrm{SSH}<-1.6 \mathrm{~m}$ in Ross Sea $\}$; boundaries are shown dotted. The upper region is $A_{\text {Weddell,SsH }}$ and the lower region is $A_{\mathrm{Ross}, \mathrm{SSH}}$.

\section{INPUT DATA AND NON-AUTONOMOUS FLOW MODEL}

Our input data is generated by the ORCA025 global ocean model [7]. In the Southern Ocean, the model grid follows a Mercator projection. Eddy characteristics of the model compare favorably with satellite and drifter observations [7]. The model is spun up for 7 years and the 8th year is used here. The available model output consists of 3-D fields of velocity averaged over 5-day intervals. The dataset (1440 points by 350 by 45 levels) is reduced by averaging the velocities onto a $1 / 2^{\circ}$ grid using the method of Aumont et al. [8] that preserves the divergence accurately.

Figure 1 shows a 60-day mean sea surface height (SSH) field from the model. Under a hydrostatic and geostrophic approximation, surface flow fields follow contours of constant SSH. However, transient eddies and ageostrophic currents such as Ekman transport are not detected by a time-mean SSH field, yet they potentially contribute to particle transport. Thus, we cannot rely on $\mathrm{SSH}$ alone to accurately describe ocean flow pathways and coherent structures or areas of low dispersion.

We denote the portion of ocean south of $36^{\circ} \mathrm{S}$ by $X$; see Figure 1. As we only consider surface flow, we work on a cylinder with $X \subset \mathfrak{X}=S^{1} \times\left[-76^{\circ},-36^{\circ}\right]$ where $S^{1}$ denotes a circle parameterised from $-180^{\circ}$ to $+180^{\circ}$. We remark that the methods we describe here work equally well in three dimensions. Considered as a non-autonomous dynamical system, the ocean flow may be described by $(x, t, \tau) \mapsto \Phi(x, t ; \tau)$, where $\Phi: X \times \mathbb{R} \times \mathbb{R} \rightarrow X$ and $\Phi(x, t ; \tau)$ is the terminal point in $X$ of a trajectory beginning at $x \in X$ at time $t$ and flowing for $\tau$ time units. A trajectory $x(t):=\Phi\left(x_{0}, t_{0} ; t\right)$ is a solution to the non-autonomous ODE $\frac{d x}{d t}=f(x(t), t)$ with initial condition $x\left(t_{0}\right)=\Phi\left(x_{0}, t_{0} ; 0\right)$. The vector field $f: X \times \mathbb{R} \rightarrow \mathbb{R}^{2}$ is obtained from the output of the ORCA025 model.

\section{ALMOST-INVARIANT SETS, COHERENT STRUCTURES, AND TRANSFER OPERATORS}

We will say that a set $A \subset X$ is $\Phi$-invariant over $[t, t+\tau]$ if $A=\Phi(A, t+s ;-s))$ for all $0 \leq s \leq \tau$. Coherent structures obey an approximate invariance principle over short periods of time. We shall call a set $A \subset X$ almostinvariant if

$$
\rho_{t, \tau}(A):=\frac{\mu(A \cap \Phi(A, t+\tau ;-\tau))}{\mu(A)} \approx 1,
$$

where $\mu$ is the probability measure with density $\Gamma(\theta, \phi)=$ $\mathcal{N} \cos \phi$ and $\mathcal{N}$ is a normalization factor so that $\int_{X} \Gamma(\theta, \phi) d \theta d \phi=1$. The measure $\mu$ has the property that $\mu([a, b] \times[c, d])$ is the area of the region $[a, b] \times[c, d]$ on the curved surface of the Earth, and is a natural reference measure for quantifying almost-invariance. The ratio in (1) is the proportion of the set $A$ that remains in $A$ at time $t+\tau$ under the flow from time $t$ to time $t+\tau$. Clearly, the closer this ratio is to unity, the closer the set $A$ is to being $\Phi$-invariant over $[t, t+\tau]$. In order to discover coherent structures in the flow $\Phi$, we seek to find dominant almost-invariant sets.

The notion of almost-invariant sets arose as a means of discovering dominant geometric structures in general dynamical systems [9] and has been refined and applied in a variety of settings, e.g. [10-12]. In order to locate these almost-invariant sets we introduce a transfer operator describing flows for short periods. This transfer operator approach has been validated in a number of autonomous systems $[11,13]$ and on the periodically forced double-gyre flow [4], where we verified that the almostinvariant sets obtained precisely describe the two gyres defined by the flow [13].

We define a linear operator $\mathcal{P}_{t, \tau}: L^{1}(X, m) \circlearrowleft$ by

$$
\mathcal{P}_{t, \tau} g(x)=\frac{g(\Phi(x, t+\tau ;-\tau))}{|\operatorname{det} D \Phi(\Phi(x, t+\tau ;-\tau), t ; \tau)|},
$$

where $m$ is normalised Lebesgue measure on $X$. If there is a $\Phi$-invariant set $A \subset X$ over $[t, t+\tau]$, then $\mathcal{P}_{t, \tau}(\Gamma$. $\left.\chi_{A}\right)=\Gamma \cdot \chi_{A}$. Thus $\Gamma \cdot \chi_{A}$ is an eigenfunction of $\mathcal{P}_{t, \tau}$ with eigenvalue 1 . Sets $A$ that are almost-invariant correspond to eigenfunctions of $\mathcal{P}_{t, \tau}$ with real eigenvalues very close to $1[9]$.

To access these eigenfunctions numerically, we construct a finite-dimensional Galerkin approximation of $\mathcal{P}_{t, \tau}$ based on a fine partition $\left\{B_{1}, \ldots, B_{n}\right\}$ of $X$. This approach is due to a suggestion of Ulam [14] in the context of discrete time maps of the unit interval. Following 
Ulam's approach we form the transition matrix

$$
\mathbf{P}_{t, \tau ; i, j}=\frac{m\left(B_{i} \cap \Phi\left(B_{j}, t+\tau ;-\tau\right)\right)}{m\left(B_{i}\right)} .
$$

The matrix $\mathbf{P}_{t, \tau}$ is stochastic. The entry $\mathbf{P}_{t, \tau ; i, j}$ may be interpreted as the probability that a point selected uniformly at random in $B_{i}$ at time $t$ will be in $B_{j}$ at time $t+\tau$.

\section{NUMERICAL IMPLEMENTATION}

\section{A. Oceanic domain and discretization}

Our domain $X$ is defined by $X=\{x \in \mathfrak{X}$ : $\left.\left\|f\left(x, t_{0}\right)\right\|_{2}>10^{-6} \mathrm{~m} / \mathrm{s}\right\}$ where $t_{0}$ denotes January 1 . We think of $X$ as $\mathfrak{X}$ with the continents and islands removed. We create an approximate partition $\left\{B_{1}, \ldots, B_{n}\right\}$ of $X$ via a uniform grid of $n=24534$ boxes in longitudelatitude coordinates. Each box has side lengths $0.7^{\circ}$ degrees longitude and $0.7^{\circ}$ latitude.

To calculate $\mathbf{P}_{t, \tau}$ in practice, each partition element $B_{i}, i=1, \ldots, n$ is filled with $N$ uniformly distributed test points $y_{i, \ell} \in B_{i}, \ell=1, \ldots, N$. In the experiments reported here, $N=400$. Experiments with $N=100$ showed no appreciable difference in results. For each $i=1, \ldots, n$ we calculate $\Phi\left(y_{i, \ell}, t ; \tau\right), \ell=1, \ldots, N$ by numerical integration and set

$$
\mathbf{P}_{t, \tau ; i, j} \approx \frac{\#\left\{\ell: y_{i, \ell} \in B_{i}, \Phi\left(y_{i, \ell}, t ; \tau\right) \in B_{j}\right\}}{N}
$$

The box-discretization of $X$ and the construction of $\mathbf{P}_{t, \tau}$ is carried out efficiently using the software package GAIO $[15]$.

\section{B. Trajectory integration}

Calculation of $\Phi\left(y_{i, \ell}, t ; \tau\right)$ is carried out using a standard Runge-Kutta approach with stepsize of 1 day. Velocity field values for $x$ lying between grid points are affinely interpolated independently in the longitude and latitude directions. The velocity field $f(x, t)$ for $t$ between 5-day grid points is produced by linear interpolation. We have chosen a stepsize of 1 day as in one integration step the vast bulk of trajectories will flow only to a neighbouring grid set in the $1 / 2^{\circ}$ degree grid upon which the velocity field is defined. Since $f(x, t)$ is affine between grid points, the numerical integration error should be small.

We wish to capture a snapshot of the coherent structures at an initial time $t_{0}$. For this reason, $\tau$ should be small, however, $\tau$ should be large enough that the Lagrangian dynamics play a role. The box discretisation has the effect of a finite-range diffusion with range of the order of the box edge lengths. We wish to make $\tau$ large enough so that the advective transport dominates any discretisation-induced diffusive transport. Thus, $\tau$ should be large enough so that most trajectories leave their initial box. In the calculations reported here we choose $\tau=60$ days; on average trajectories flow $5.8^{\circ}$ of longitude and $1.5^{\circ}$ of latitude over this period. An analysis of seasonal differences can be carried out by performing a second calculation with $t_{0}$ six months later. Seasonal to annual circulation analyses could be carried out by increasing $\tau$. We limit our analysis here to the summertime months of January and February.

\section{Eigenvalue and eigenfunction calculation}

Define

$$
p_{i}=\frac{\text { Area of } B_{i}}{\text { Area of } \mathcal{B}}
$$

where $\mathcal{B}:=\bigcup_{i=1}^{n} B_{i}$. Let $A=\bigcup_{i \in \mathcal{I}} B_{i}$ with $\mathcal{I} \subset$ $\{1, \ldots, n\}$. Then it is straightforward to show [11]

$$
\rho_{t, \tau}(A) \approx \frac{\sum_{i, j \in \mathcal{I}} p_{i} \mathbf{P}_{t, \tau ; i, j}}{\sum_{i \in \mathcal{I}} p_{i}}
$$

compare with equation (1). The expression (6) is very close to equality and in the limit as $n \rightarrow \infty$ and the diameter of the boxes $\left\{B_{i}\right\}_{i=1}^{n}$ approaches zero, one obtains equality.

The vector $p$ would be an approximate fixed left eigenvector of $\mathbf{P}$ if the measure $\mu$ from Section III were $i n$ variant under $\Phi$ (formally, for each $A \subset X$ and $\tau \geq 0$, $\mu(\Phi(A, t ;-\tau))=\mu(A))$. However, $\mu$ is not strictly invariant under $\Phi$ because of (i) upwelling and downwelling, (ii) convective overturning, although the mass flux associated with convection is zero in hydrostatic models such as that used here, and (iii) the existence of trajectories that begin in $X$, but leave $X$ via the northern boundary after 2 months. We therefore perform some preprocessing[17] on $\mathbf{P}$ to ensure that $\mathbf{P}$ is stochastic and has $p$ as an exact fixed left eigenvector; that is $p \mathbf{P}_{t, \tau}=p$ for all $t, \tau$. We now transform the matrix $\mathbf{P}_{t, \tau}$ into a "time symmetric" matrix $R_{t, \tau}$ via

$$
R_{t, \tau ; i, j}=\left(\mathbf{P}_{t, \tau ; i, j}+\frac{p_{j} \mathbf{P}_{t, \tau, j, i}}{p_{i}}\right) / 2,
$$

The matrix $R$ is stochastic, has $p$ as a fixed left eigenvector, and satisfies important maximization properties related to almost-invariance [12]. Denote by $\lambda_{2}$ the second largest eigenvalue of $R$. For $A$ as above, we are guaranteed [12] that

$$
1-\sqrt{2\left(1-\lambda_{2}\right)} \leq \max _{0 \leq m(A) \leq 1 / 2} \rho_{t, \tau}(A) \leq \frac{1+\lambda_{2}}{2}
$$

As in [12] we use the right eigenvectors $v^{(k)}$ of $R$ to detect almost-invariant sets, extracting almost-invariant sets from boxes with extreme values of $v^{(k)}$. That is, 


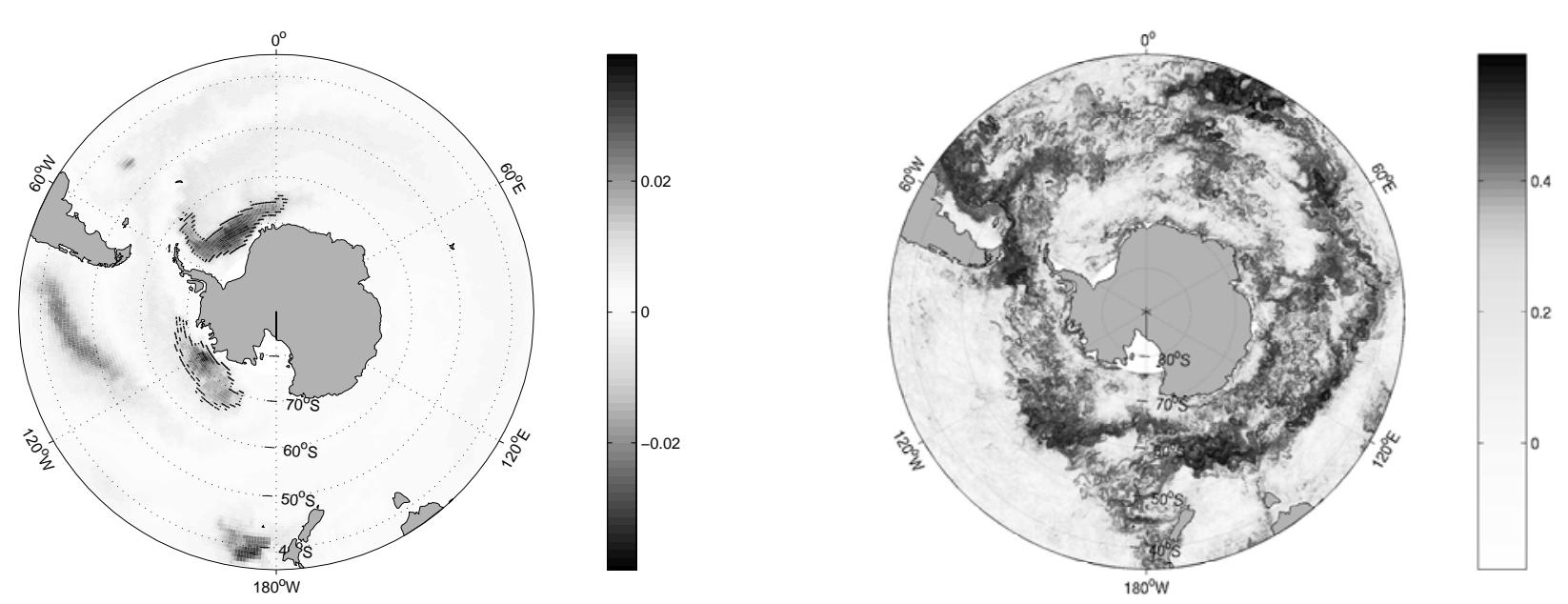

FIG. 2: The ninth eigenvector $v^{(9)}$ calculated from a 60 day flow. Coherent surface structures are highlighted in the Weddell and Ross Seas. Define regions $A_{\text {Weddell }}:=\left\{v^{(9)}>0.01\right\}$ and $A_{\text {Ross }}:=\left\{v^{(9)}<-0.01\right\}$, with boundaries shown dotted. The upper region is $A_{\text {Weddell }}$ and the lower region is $A_{\text {Ross }}$.

$A=\bigcup_{v_{i}^{(k)}>c} B_{i}$ or $A=\bigcup_{v_{i}^{(k)}<c} B_{i}$ for some $c \in \mathbb{R}, k=$ $1, \ldots, K$.

The matrix $R_{t, \tau ; i, j}$ is typically very sparse. We are interested only in the large spectral values near to 1 . The computation of the $K \ll n$ largest eigenvalues and their corresponding eigenvectors is fast using Lanczos iteration methods.

\section{RESULTS}

We demonstrate that our method detects persistent structures in the Southern Ocean surface flow in the Weddell and Ross Seas. We computed the 20 largest eigenvalues of $R$ (ranging from $\lambda_{2}=0.928$ to $\lambda_{20}=0.888$ ) and the corresponding right eigenvectors. The ninth eigenvector $v^{(9)}$ (corresponding to $\lambda_{9}=0.905$ ) identifies two coherent structures in the Weddell and Ross Seas; see Figure 2. These surface structures are not precisely aligned with the locations of the Weddell and Ross Gyres as defined by the SSH calculations shown in Figure 1. Indeed, there is a significant difference in the Ross Sea, confirming that our method picks up different structures to those defined simply by the SSH field of Figure 1. The eigenvectors $v^{(2)}-v^{(8)}$ determine other coherent structures in the surface flow that are not directly related to the Weddell and Ross Gyres. Larger coherent structures correspond to more highly ranked eigenfunctions as a larger proportion of the domain is coherent. As the total area occupied by the gyres represents a relatively small coherent structure, it is the eigenvector $v^{(9)}$ that detects the gyres.

We investigate the quality of the coherence and nondispersiveness of the structures shown in Figures 1 and
FIG. 3: Estimation of the finite-time Lyapunov exponent field from 1 January-29 February. Dark colors indicate regions of high stretching. The field was constructed using 100,213 boxes and 40 points per box; see [16].

2 via equation (1). Applying (6) yields $\rho_{t_{0}, \tau}\left(A_{\text {Weddell }}\right)=$ 0.91 versus $\rho_{t_{0}, \tau}\left(A_{\text {Weddell,SSH }}\right)=0.80$ and $\rho_{t_{0}, \tau}\left(A_{\text {Ross }}\right)=$ 0.85 versus $\rho_{t_{0}, \tau}\left(A_{\mathrm{Ross}, \mathrm{SSH}}\right)=0.75$. The calculation $\rho_{t_{0}, \tau}\left(A_{\text {Weddell }}\right)=0.91$ (for example), states that $91 \%$ of the surface mass in $A_{\text {Weddell }}$ remains (is trapped) in $A_{\text {Weddell }}$ at time $t_{0}+\tau$. Thus the above calculations demonstrate that the regions detected by the transfer operator approach are more coherent over the 60 day period considered than those determined by sea surface height. Such information is very useful for surface larval drift and biomass transport models which might otherwise have been assumed to be governed by gyres in positions defined by the SSH via a geostrophic assumption.

To compare our new method with finite-time Lyapunov exponents, we approximated the FTLE field for the same 60 day period; see Figure 3. As peaks in the FTLE field are associated with barriers to transport [4], we may expect that non-dispersive regions show up as pale regions surrounded by dark regions in Figure 3. There is some evidence of this in the Weddell and Ross Seas, but these regions are not identified nearly as clearly as in Figure 2. Further work will extend the transfer operator analysis to the coherent structures of global-scale three-dimensional ocean flow.

\section{Acknowledgments}

GF is partially supported by an ARC Discovery Project and a UNSW Faculty of Science Research Grant, and KP by the UPB Research Award 2006 and by the DFG GK-693 of the Paderborn Institute for Scientific Computation. MHE is supported by an ARC Federation Fellowship and AMT participation by an ARC Linkage International Award. We thank Martin Krüger for assis- 
[1] L.-L. Fu, Geophys. Res. Lett. 33, L14610 (2006).

[2] G. Haller, Chaos 10, 99 (2000).

[3] G. Haller, Phys. Fluids 14 (2002).

[4] S. Shadden, F. Lekien, and J. Marsden, Physica D 212, 271 (2005).

[5] S. Wiggins, Annu. Rev. Fluid Mech. 37, 295 (2005).

[6] B. Joseph and B. Legras, J. Atmos. Sci. 59, 1198 (2002).

[7] B. Barnier, G. Madec, T. Penduff, J. Molines, A. Treguier, J. L. Sommer, A. Beckmann, A. Biastoch, C. Boening, J. Dengg, et al., Ocean Dynamics (2006).

[8] O. Aumont, J. Orr, D. Jamous, P. Monfray, O. Marti, and G. Madec, Clim. Dynam. 14, 101 (1998).

[9] M. Dellnitz and O. Junge, SIAM J. Numer. Anal. 36, 491 (1999).

[10] M. Dellnitz, O. Junge, W. Koon, F. Lekien, M. Lo, J. Marsden, K. Padberg, R. Preis, S. Ross, and B. Thiere, Int. J. Bifur. Chaos 15, 699 (2005).
[11] G. Froyland and M. Dellnitz, SIAM J. Sci. Comput. 24, 1839 (2003).

[12] G. Froyland, Physica D 200, 205 (2005).

[13] G. Froyland, K. Padberg, In preparation (2007).

[14] S. Ulam, A collection of mathematical problems (Interscience Publishers, 1960).

[15] M. Dellnitz, G. Froyland, and O. Junge, in Ergodic Theory, Analysis, and Efficient Simulation of Dynamical Systems, edited by B. Fiedler (Springer, 2001), pp. 145174.

[16] K. Padberg, PhD thesis, Universität Paderborn (2005).

[17] We will elaborate upon this preprocessing in a future publication. We remark that while the preprocessed matrix $\mathbf{P}$ was used to identify the almost-invariant structures, the evaluation of these structures as reported in Section V via (6) used the "raw" matrix $\mathbf{P}$. 